\title{
Mixed SOC diffusive dynamics as a paradigm for transport in fusion devices
}

\author{
R. Sánchez ${ }^{\mathrm{a}}$, D.E. Newman ${ }^{\mathrm{b}}$, B.A. Carreras ${ }^{\mathrm{c}}$ \\ ${ }^{a}$ Universidad Carlos III de Madrid, Leganés, Madrid, Spain \\ b University of Alaska - Fairbanks, Fairbanks, Alaska, United States of America \\ ${ }^{c}$ Oak Ridge National Laboratory, Oak Ridge, Tennessee, United States of America
}

\begin{abstract}
A recently proposed paradigm for understanding turbulent transport dynamics in magnetically confined plasmas, based on the concept of self-organized criticality (SOC), is extended to include the interplay among the many transport mechanisms existing in real plasmas. This extended model might shed some light on the experimentally observed violation at fluctuation scales of the scale invariance condition inherent to the standard SOC model. At the same time, it might provide new experimental tests that could help to validate the possible relevance of the SOC model for understanding turbulent plasma dynamics, since some computations based on a cellular automata realization of such an extended model reveal a dramatic change in the dynamics, with the edge coming to play a dominant role. This might give some hints towards understanding the experimental fact relating good plasma core confinement to a good conditioning of the edge within an SOC paradigm.
\end{abstract}

\section{Introduction}

In the past ten years there has been a great deal of interest in the concept of self-organized criticality (SOC) [1] as a unifying explanation for some of the observed, apparently universal, dynamics of complex systems. This concept brings together ideas about non-linear self-organization with the often observed near-critical behaviour of many natural phenomena. In an SOC system, the non-linear dynamics in the presence of a drive organizes the system to fluctuate around a state marginal to major disruptions. This steady state becomes a stable fixed point for the dynamics, which is therefore reached without any need for external tuning (which accounts for the selforganized part of its name), being thus very resistant to external perturbations. When at this fixed point, the system displays the same spatial and temporal self-similar spectra characteristic of critical points in phase transition theory (which explains the other half of the name). These spectra have been shown to exist in remarkably similar forms in a wide variety of physical and biological systems [2-6]. The transport out of the SOC system, which must balance the incoming drive to reach the steady state, is driven by avalanches. These can have all sizes and durations (limited only by the size and life of the system), and their probability distribution functions (PDFs) are well described by power laws. Key ingredients of SOC systems are: (a) an instability threshold; (b) two disparate timescales, one associated with the drive and the other with the instability relaxation. It is easy to find candidates for these ingredients in a confined plasma. With this in mind, some modes (such as the ITG mode at the core and resistive interchange or ballooning modes at the edge) have been proposed as candidates for driving a significant amount of avalanche transport while remaining subcritical on average, with the plasma behaving as an SOC system. Such a paradigm could help us to understand many experimental results not properly accounted for by other transport models [7-10]:

(a) The strong transport measured even when the profiles are on average stable with respect to the suspected responsible instability;

(b) The scaling of confinement time in L mode discharges with the system size;

(c) The decorrelation of avalanches by a sheared flow and the change in scaling of the confinement time in enhanced confinement modes;

(d) The existence of universal indexes in measured broadband spectra;

(e) The experimental finding of canonical plasma profiles.

Some experimental evidence consistent with the SOC assumption has already been reported. Results of the analysis of edge electrostatic fluctuation data from several experiments, including tokamaks, stellarators and reverse field pinches, showed their selfsimilar character down to timescales of the order of ten times the turbulence decorrelation time. At the same time, some strong evidence for the existence of radial correlations over distances longer than 


\section{R. Sánchez et al.}

the turbulence correlation length was also found [9]. Experiments supporting the radial propagation of avalanche-like events have also been recently reported in the DIII-D tokamak [11]. But, at the same time, some groups have reported small deviations of the strict self-similarity hypothesis that underlies the SOC paradigm when going down to the scale of fluctuations. These deviations are detected in the form of some degree of intermittency, which manifests itself as a departure from a Gaussian distribution in the PDF tails of the fluctuations $[12,13]$. These small scale invariance violations are, however, to be expected since a number of the simultaneously active transport mechanisms that drive continuous subdominant flow out of the plasma could break scale invariance $[14,15]$.

To study this interaction, we will divide the outcoming flux into two contributions:

(a) A dominant discontinuous flux, which will be referred to as the avalanche or SOC channel, to which any subcritical turbulence driven flux will contribute;

(b) A subdominant continuous flux, which will be referred to as the diffusive channel, fed by any collision driven flux or supercritical turbulence driven flux.

Notice that we would expect the transport to lack any characteristic scale when dominated by the avalanche channel, while it would show the characteristic scales of the underlying physical mechanism in the other case. We will then show, using a simpler cellular automata realization of such a model, that the interaction between this dominant SOC channel and the ever present diffusive channel is far from negligible, even for small diffusivity, efficiently erasing certain ranges of scales that will break scale invariance, leaving traces that should be experimentally detectable. The article is organized as follows. In Section 2, the sandpile cellular automata used in the study are introduced. In Section 3, the changes in the statistical properties of the sandpile avalanche transport for increasing diffusivity are described. Special attention is paid to the effect that diffusivity has on the power spectrum of the transport fluctuations and on the existence of long term correlations in the system, since these are considered to be characteristic properties of the standard SOC paradigm. The change of the role played by the edge in the dynamics is explored in Section 4. Finally, some conclusions relative to the application of this extended model to fusion plasmas are drawn in Section 5 .

\section{Sandpile cellular automata model}

To identify the characteristic traces of the interplay between these transport channels we turn to a simpler model, that of a standard running sandpile $[16,17]$. Sandpiles have been frequently used to study the underlying physics aspects of SOC dynamics for their easy implementation as cellular automata and the possibility of obtaining long records of data amenable to exhaustive statistical analysis. In addition, because the analogies with turbulent plasma transport models are clear, as argued in Ref. [7], with each cell representing a turbulent eddy, the critical sandpile gradient representing the local instability threshold and the random rain of sand playing the role of background or heating noise. However, it is important to keep in mind that differences are also numerous, essentially arising from the uniform parameter dependence assumed, the discrete and fixed size of turbulent profile relaxation (the $N_{F}$ parameter, which will be introduced later) and the lack of any effects from parallel and/or poloidal dynamics, such as those induced by radial electric fields or parallel flows. However, even with all these shortcomings, the fundamental physical aspects of the interaction between the two transport channels are well captured by the sandpile realization of SOC dynamics.

To include both transport channels, we have added to the standard sandpile a diffusive component that can interact with varying relative intensity with the dominant avalanche-like component. The SOC character of the sandpile dynamics arises from the existence of a critical slope $\left|Z_{c}\right|$, which when locally overcome gives rise to the removal of the excess sand to adjacent positions. The sandpile domain is divided into $L$ cells. In each of them, labelled by the index $n$ with $0<n \leq L$, the amount of sand is $h_{n}$. The sandpile state evolves as follows. First, a grain of sand is added randomly to the cells with probability $P_{0}$. Next, all the cells are checked for stability by comparing the local gradient $Z_{n}$ with the critical gradient. Finally, the cells are time advanced, with the unstable cells overturning and moving a prescribed amount of sand $N_{f}$ to the next cell: $h_{n}=h_{n}-N_{f}$ and $h_{n+1}=h_{n+1}+N_{f}$. Typical values in the simulations are $L=400, Z_{c}=200$ and $N_{f}=30$. The boundary condition is open at $x=L$ (particles that reach there are lost) and closed at $x=0$ in order to allow simulation of only half of the entire pile. To this standard automata model we have added a continuous diffusive component in the following way: a diffusive flux 
at the $n$th cell is computed as $\Gamma_{n}=D_{0}\left(Z_{n+1}-Z_{n}\right)$, with the flux going to the $(n+1)$ th cell when positive and to the $(n-1)$ th cell otherwise.

By assuming a spatially uniform rain of sand, the sandpile domain will be split into two distinct subdomains. In the subdomain extending inwards from the boundary at $x=0$, most of the transport will go through the diffusive channel (due to the closed boundary condition imposed at $x=0$ ). In the second subdomain, which extends inwards from the boundary at $x=L$, transport will be mainly driven by avalanches. Both subdomains will be linked by a crossover region, where both channels have similar intensities. Even with its apparent simplicity, this subdomain disposition somewhat resembles some of those found in low power ohmic or electron cyclotron heated plasma profiles, where gyro-Bohm transport seems to apply in the core with strongly subthreshold temperature and density profiles, while the edge transport seems to be much more Bohm-like, with profiles having slopes closer to their local threshold values.

In order to estimate analytically the location of the crossover point, we assume that the diffusive channel is always subdominant when the avalanchelike channel is locally present. In this way, the crossover region collapses into a single point, since the turbulent channel dominates as soon as $\left|Z_{n}\right| \geq$ $\left|Z_{c}\right|$. The location of the crossover point is easily estimated from a simple analytical model,

$$
\frac{\partial h}{\partial t}=D_{0} \frac{\partial^{2} h}{\partial x^{2}}+\theta\left(\left|\frac{\partial h}{\partial x}\right|-\left|Z_{c}\right|\right) V \frac{\partial h}{\partial x}+S(x)
$$

with the source equal to the average local influx $P_{0}$ and with $V=\left|Z_{c}\right| / P_{0}$ determined by matching the two solutions at the transition point. The onoff character of the non-linearity is modelled using a Heaviside function $\theta(x)$, which will make the nonlinearity vanish below the critical gradient. The transition point is found to be

$x_{t}^{*} \equiv x_{t} / L=\left(\left|Z_{c}\right| / L\right)\left(D_{0} / P_{0}\right)$

which agrees well with sandpile simulations. The solutions for the sandpile profile on both subdomains are

$n=-\frac{P_{0} L^{2}}{2 D_{0}} x^{* 2}+Z_{c} L-\frac{Z_{c}^{2} D_{0}}{2 P_{0}}$

for $0 \leq x^{*}<x_{t}^{*}$ and

$n=\left|Z_{c}\right| L\left(1-x^{*}\right)$

for $x_{t}^{*}<x^{*} \leq 1$ with the marginal SOC profile for $x^{*}>x_{t}^{*}$ and a parabolic diffusive profile for $x^{*}<x_{t}^{*}$, which is a good approximation to the actual simulated profiles. This subdomain structure is maintained if the condition $x_{t}^{*}<1$ holds. In terms of the free parameters of the simulation this requires $D_{0} / P_{0}<L / Z_{c}$, which sets an upper limit for the diffusivity ratio $D_{0} / P_{0}$. The crossover point will move inwards (outwards) for increasing (decreasing) drive or decreasing (increasing) diffusivity. At the same time, it is straightforward to see that Eq. (2) is approximately equal to the fraction of the total average transport leaving the sandpile through the diffusive channel. Therefore, the average ratio of the intensities of both channels can also then be tuned by varying $D_{0} / P_{0}$, for fixed $\left|Z_{c}\right|$ and $L$.

\section{Statistical properties of diffusive sandpile avalanche transport}

The total transported sand leaves the sandpile through either the diffusive or the SOC channel. Although it is clear from the discussion at the end of the previous section that the part of the transport going through the diffusive channel will increase as the diffusivity ratio increases, in most fusion plasmas it will never become even comparable to that in the turbulent channel. Therefore, we will be interested in looking at the change in the properties of avalanche transport when a small subdominant diffusive transport regime is present. We can stay in that regime by requiring $D_{0} / P_{0} \ll 1<L / Z_{c}$. To characterize avalanche transport we will use the standard tools used to study the pure SOC case:

(a) Look for occurrences of violations of scale invariance,

(b) Calculate universal indexes in the power spectra of turbulent transport fluctuations,

(c) Look for long term correlations in the autocorrelation function of the fluctuations or via the range statistics rescaled analysis.

\subsection{Break-up of scale invariance in avalanche transport}

The change in the properties of avalanche transport when the diffusivity ratio is increased from zero (the standard SOC case) is well illustrated in Fig. 1(a). This figure shows a time trace of $g(t)$, whose time series is formed by calculating at each 


\section{R. Sánchez et al.}

iteration the total number of overturning (i.e. unstable) sites. It is, therefore, a measure of the avalanchelike activity taking place within the system. For the pure SOC case, the time variation of this function is strongly bursty, showing scale invariance up to system scales, with events of all possible sizes and durations. In contrast, for increasing diffusivity ratio (Fig. 1(b)), the time history of $g(t)$ becomes a more intermittent constant size signal. The signal transition from burstiness to intermittency is probably caused by the continuous smoothing of the local inhomogeneities in the sandpile slope profile by subdominant diffusion, which makes it increasingly difficult for avalanches to take place. For small values of the ratio $D_{0} / P_{0}$, the subdominant diffusive component cannot, however, completely balance the source at the submarginal level, causing the slope to build up to a point at which a larger avalanche event can occur. On the other hand, the constant size of the signal can be associated with the appearance of a characteristic spatial scale. It will be shown in Section 4 that avalanches tend to be initiated very close to the edge region for sufficiently large diffusivity. At this point, continuous diffusion is able to smooth out most of the inhomogeneities in the inner regions, and the avalanche has thus no obstacles to prevent its propagation inwards, until it reaches the transition point $x_{t}$. A characteristic length of the order of $L-x_{t}$ therefore appears, which decreases with increasing values of the $D_{0} / P_{0}$ ratio according to Eq. (2).

\subsection{Power spectrum of global avalanche transport}

One of the more useful tools with which to characterize the dynamics of SOC systems is the power spectrum of the time series of $g(t)$ (as a matter of fact, the understanding of this power spectrum structure and its ubiquitous presence in nature was one of the main motivations for the initial formulation of the SOC paradigm),

$P(w)=|G(w)|^{2}$

with

$G(w)=\frac{1}{\sqrt{2 \pi}} \int_{-\infty}^{\infty} g(t) e^{-i w t} d t$.

In the pure SOC case, this spectrum can be split into several distinct regions where it follows power laws, $P(w) \propto w^{-\alpha}$, with varying universal exponents $\alpha[16,17]$. Each of these regions is related to a different type of event taking place in the system:
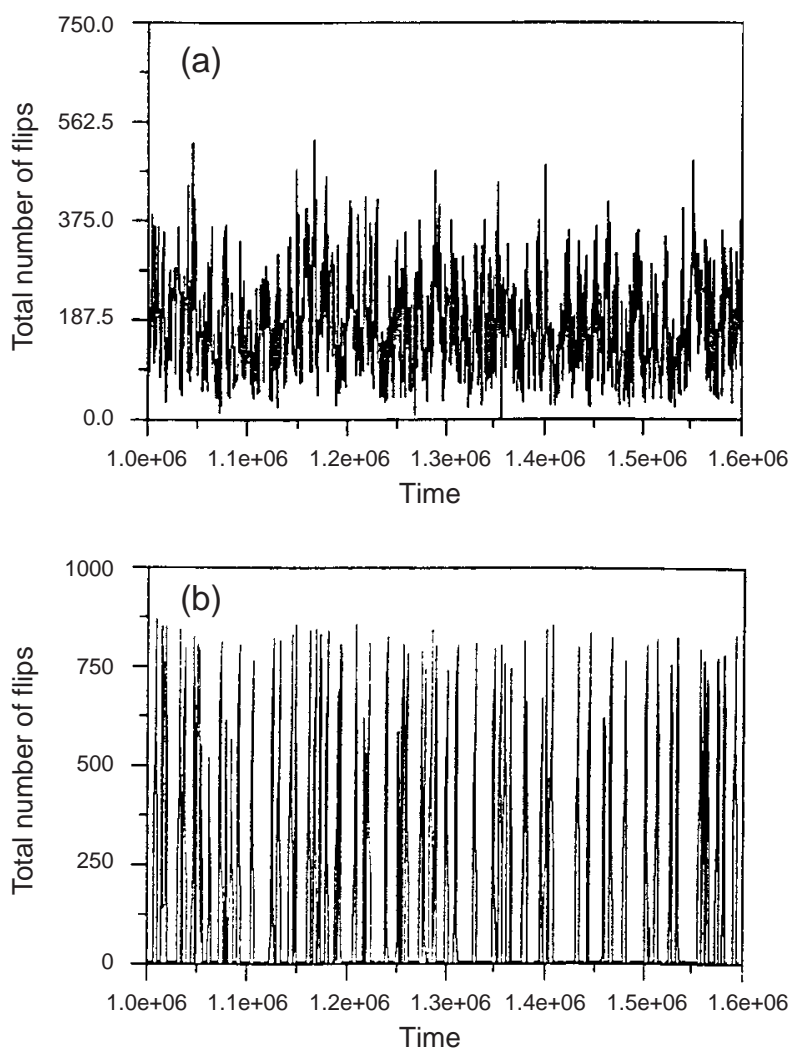

Figure 1. Time histories of $g(t)$ (defined in text): (a) pure SOC case $\left(D_{0} / P_{0}=0\right)$, (b) diffusive case $\left(D_{0} / P_{0}=1\right)$.

(a) The region at low frequencies with $\alpha=0$ corresponds to large catastrophic single events in which large amounts of stored energy are released and with characteristic scales that involve the whole system.

(b) The region at intermediate frequencies with $\alpha=1$ has been related to the overlapping of avalanches [17].

(c) Finally, for large frequencies, a region with an exponent larger than 2 exists, corresponding to small scale, and therefore frequent, events which involve very small parts of the system.

One of the ways of ascertaining to what extent (if any) the increase of diffusivity ratio modifies the system dynamics is to look for modifications in the power spectrum structure just described. It is interesting to note that for increasing diffusivity (Fig. 2) the $1 / f$ region of the spectrum begins to shrink and eventually disappears at a threshold value as low as $D_{0} / P_{0}=0.1$. Notice that, at this value, transport is still strongly dominated by avalanches. This surprising result seems to suggest that diffusion can reduce 


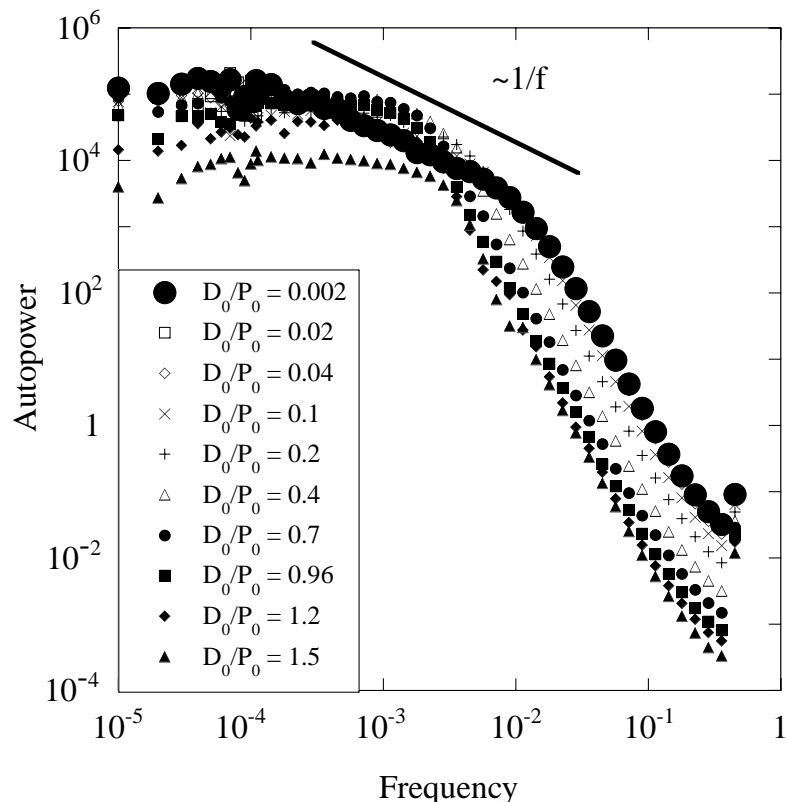

Figure 2. Power spectra of $g(t)$ for increasing values of the diffusivity ratio.

avalanche overlapping very efficiently, even at much smaller values than those needed for achieving control over global transport. The continuous smoothing of the local profile inhomogeneities and the concomitant decorrelation carried out by the diffusive process thus seem able to erase the memory of the system contained as irregularities of the slope profile, thus preventing triggering of avalanches (here, memory is understood as the existence of correlations between the previous history of the system and its future evolution). At the same time, the flat low frequency region extends to higher frequencies suggesting that, as a result of the increased diffusion, larger isolated events become more common. We will find other evidence of this behaviour later, when the edge dynamics are explored in Section 4.

\subsection{Existence of long term time correlations in global avalanche transport}

Another characteristic feature of SOC dynamics is the appearance of long range spatial and temporal correlations, closely related to the existence of the type of system memory discussed in Section 3.2. These correlations can be partly identified by studying the autocorrelation function of $g(t)$,

$\Delta(\tau)=\int_{-\infty}^{\infty} g(t) g(\tau-t) d t$

The occurrence of algebraically decaying tails in the autocorrelation function has long been used as a

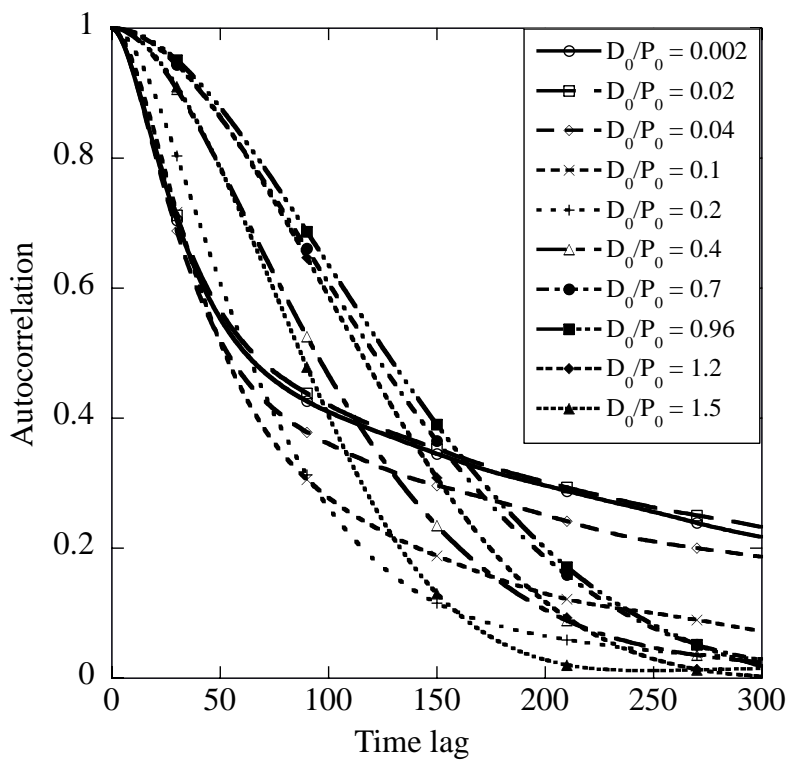

Figure 3. Autocorrelation function of $g(t)$ for increasing values of the diffusivity ratio.

proof of the existence of long term correlations in the system. For the standard SOC case, this algebraic tail is apparent, as shown in Fig. 3. However, important changes take place in the autocorrelation function behaviour when $D_{0} / P_{0}$ increases. First, the algebraic tail vanishes very quickly, for values of the diffusive ratio around $D_{0} / P_{0}=0.1$, consistent with the other diagnostics. However, at the same time, the correlation length of the fluctuations surprisingly increases beyond the threshold until the ratio becomes $D_{0} / P_{0}=1$, when it begins to decrease very quickly (Fig. 4). This behaviour can be explained by assuming again that the smaller scales are the ones which are going to be more efficiently erased by diffusion (and a value of $D_{0} / P_{0}$ just above the threshold is sufficient to carry out this task), leaving only larger scales responsible for transport events. For sufficiently large diffusion though (when $D_{0} / P_{0} \simeq 1$ ), these scales will also be affected and the correlation length again decreases.

Another method to look for long term correlations is the rescaled adjusted $R / S$ analysis method proposed by Mandelbrot and Wallis [18] based on previous work by Hurst [19]. An $R / S$ analysis of a time series is carried out in the following way. For a time series of length $n, X=X_{k}, k=1, \ldots, n$, with mean $\bar{X}_{n}$ and variance $S_{n}^{2}$, the $R / S$ ratio is computed as

$\left(\frac{R}{S}\right)_{n} \equiv \frac{\max \left(0, W_{1}, \ldots, W_{n}\right)-\min \left(0, W_{1}, \ldots, W_{n}\right)}{\left.\sqrt{(} S_{n}^{2}\right)}$ 


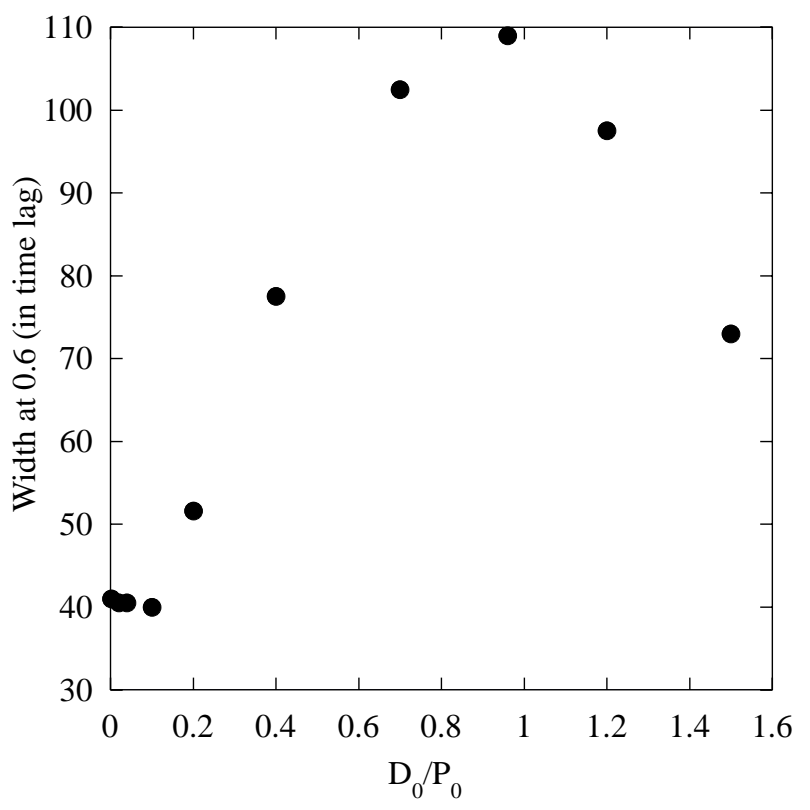

Figure 4. Correlation length of $g(t)$ for increasing values of the diffusivity ratio.

where $W_{k}=X_{1}+\ldots+X_{k}-k \bar{X}_{n}$. For some range of the time lag $n$, this ratio possibly scales as $n^{H}$, where $H$ is called the Hurst exponent. A constant value of the $H$ parameter over a long range of time lag values is a signature of the self-similarity of the signal over that range of timescales. The value of $H$, on the other hand, can unveil hidden long range dependences: $H>0.5$ implies long term correlations, $H<0.5$ reveals anti-persistence, while $H=0.5$ implies a totally random signal. In the standard SOC sandpile, a value for the Hurst exponent close to $H=0.8$ is obtained which extends for more than two decades (Fig. 5). This value of $H$ reveals the strong self-similarity of the system over this range of time lags. When the diffusivity ratio is increased, the situation changes consistently with the power spectrum results shown in Section 3.2: the range over which the $R / S$ ratio scales linearly begins to decrease very quickly, eventually ceasing to exist above the same threshold value, $D_{0} / P_{0} \sim 0.1$, that precluded the existence of the $1 / f$ region. This shrinkage signals the appearance of characteristic scales in the dynamics. Simultaneously, the value of the Hurst exponent decreases, which means that the memory of the system stored in the local inhomogeneities is erased by the continuous smoothing driven by diffusion.

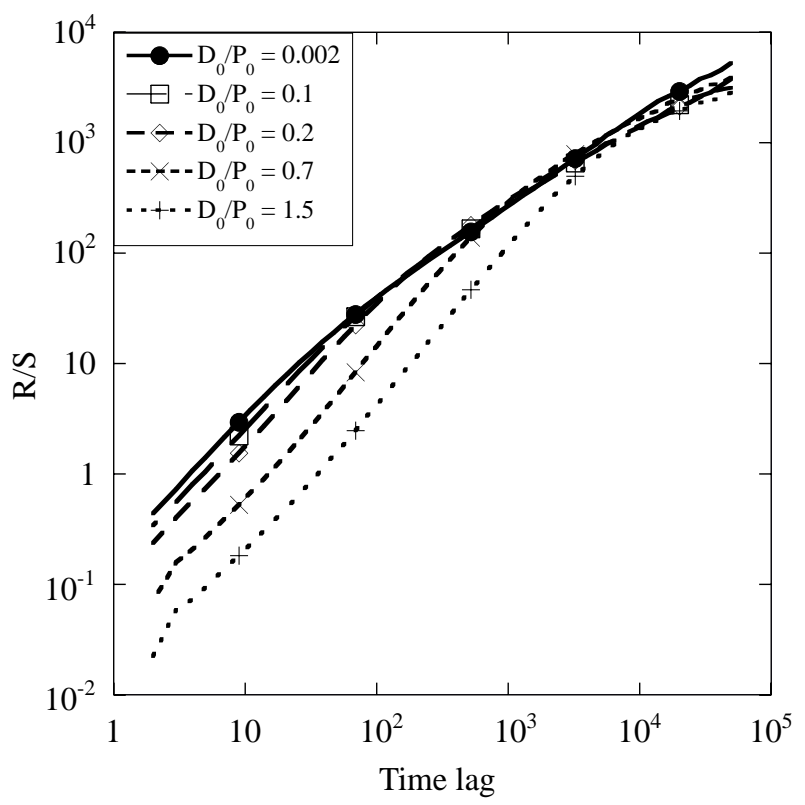

Figure 5. $R / S$ analysis of $g(t)$ for increasing values of the diffusivity ratio.

\section{Importance of the edge in SOC diffusive dynamics}

The central role that the edge comes to play in SOC diffusive dynamics is revealed by the change in the probability distribution functions (PDFs) of the sandpile locations where avalanche are initiated. In Figs 6(b) and (d) this PDF is shown for the standard SOC sandpile. Notice that two types of avalanche are distinguished: (a) 1-D avalanches, which involve the propagation of a hole up the slope, or the propagation down the slope of a bump; (b) 2-D avalanches, for which a hole and a bump simultaneously propagate (the reason for their different 'dimensionalities' is easily understood by looking at Fig. 7, where the time evolution of the sandpile unstable sites is shown). It is important to distinguish them since 2-D avalanches are the ones carrying most of the transport out of the pile. In the standard sandpile, both PDFs are rather flat, since any location has roughly the same probability of becoming unstable due to the uniform rain of sand. However, if we calculate the same PDFs for the diffusive case (see Figs 8(b) and (d) for the case with $D_{0} / P_{0}=1$ ), the PDF for the 2-D avalanches becomes strongly peaked at the edge, implying that most large transport events are now triggered from the edge. In this case, it is interesting to look also at the PDF of the locations where avalanches are terminated when moving up the slope, since it will tell us how deep within 
Article: Mixed SOC diffusion dynamics
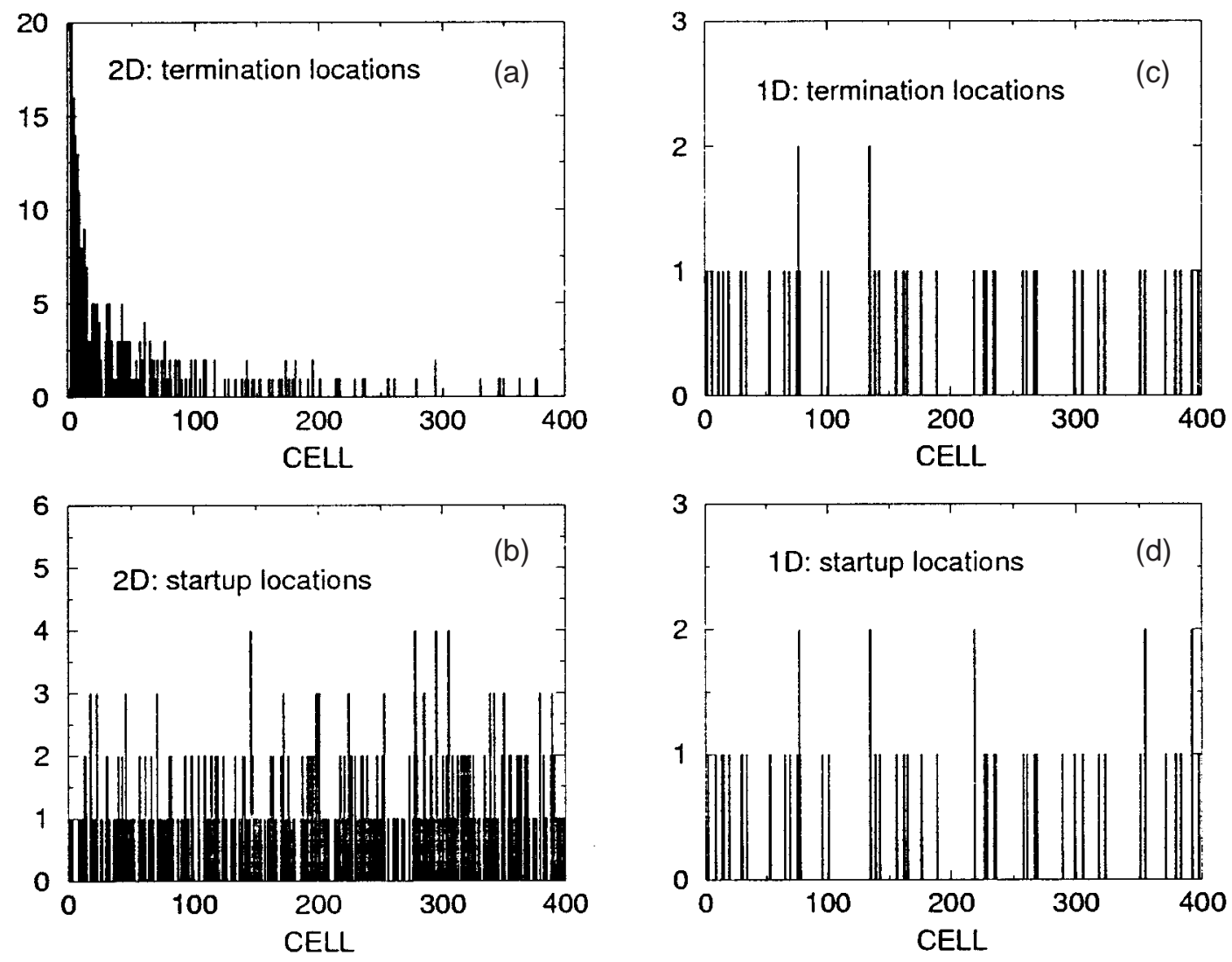

Figure 6. (b, d) PDF of avalanche startup and (a, c) termination points for the pure sandpile SOC case $\left(D_{0} / P_{0}=0\right)$.

the pile the dynamics will be sensitive to what is happening at the edge (notice that in the standard SOC case, with evenly distributed initial points, this PDF does not provide very useful information). Figures 8(a) and (c) clearly show how these PDFs are strongly peaked at the crossover point $x_{t}$ for 2 -D avalanches. Interestingly, the PDF for 1-D avalanches is very similar to that of the standard sandpile, but extended only throughout the SOC region. All these results seem to suggest that in SOC diffusive systems most large avalanches are triggered at the edge, and then propagate all the way through the SOC region. The physical explanation for this behaviour is found in the higher average slope at the edge, which is a result of the boundary condition imposed at $x=L$. Owing to the instantaneous removal of sand from the last cell, some allowed slope reduction transitions are not possible at the edge. For instance, the slope can be reduced by -1 if a grain of sand happens to fall into the next cell. However, at the edge, this grain will be removed instantaneously. For the same reason, the edge slope can only be reduced by
$-N_{f}$ when unstable, while at any other location it would be reduced by $-2 N_{f}$. In the SOC case, this steeper edge region only involves the last two points, but it expands inwards to accommodate the outgoing flux for increasing diffusivity. As a result, the edge becomes the most probable location for initiating avalanches, which, because of the previous diffusive smoothing of the inner slope profile, will find no obstacle to propagation across all of the SOC region. Note that this is the physical mechanism that introduces the characteristic length $L-x_{t}$ discussed in Section 3.

\section{Conclusions}

As already mentioned in Section 1, it has been recently proposed that plasma transport (at least in L mode discharges) might be dominated by a subcritical turbulent mechanism whose intensity (and even its nature) could change from one radial location to another. If this were the case, we would expect the 


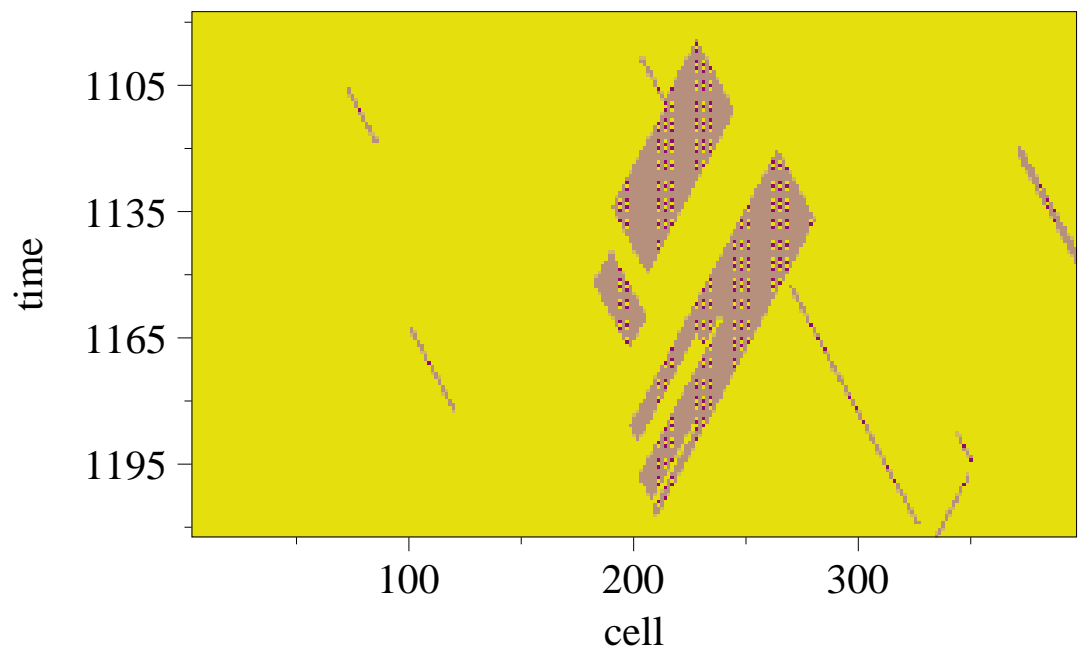

Figure 7. Time evolution of the sandpile slope (the horizontal scale corresponds to location and the vertical scale to time) for a standard SOC sandpile.
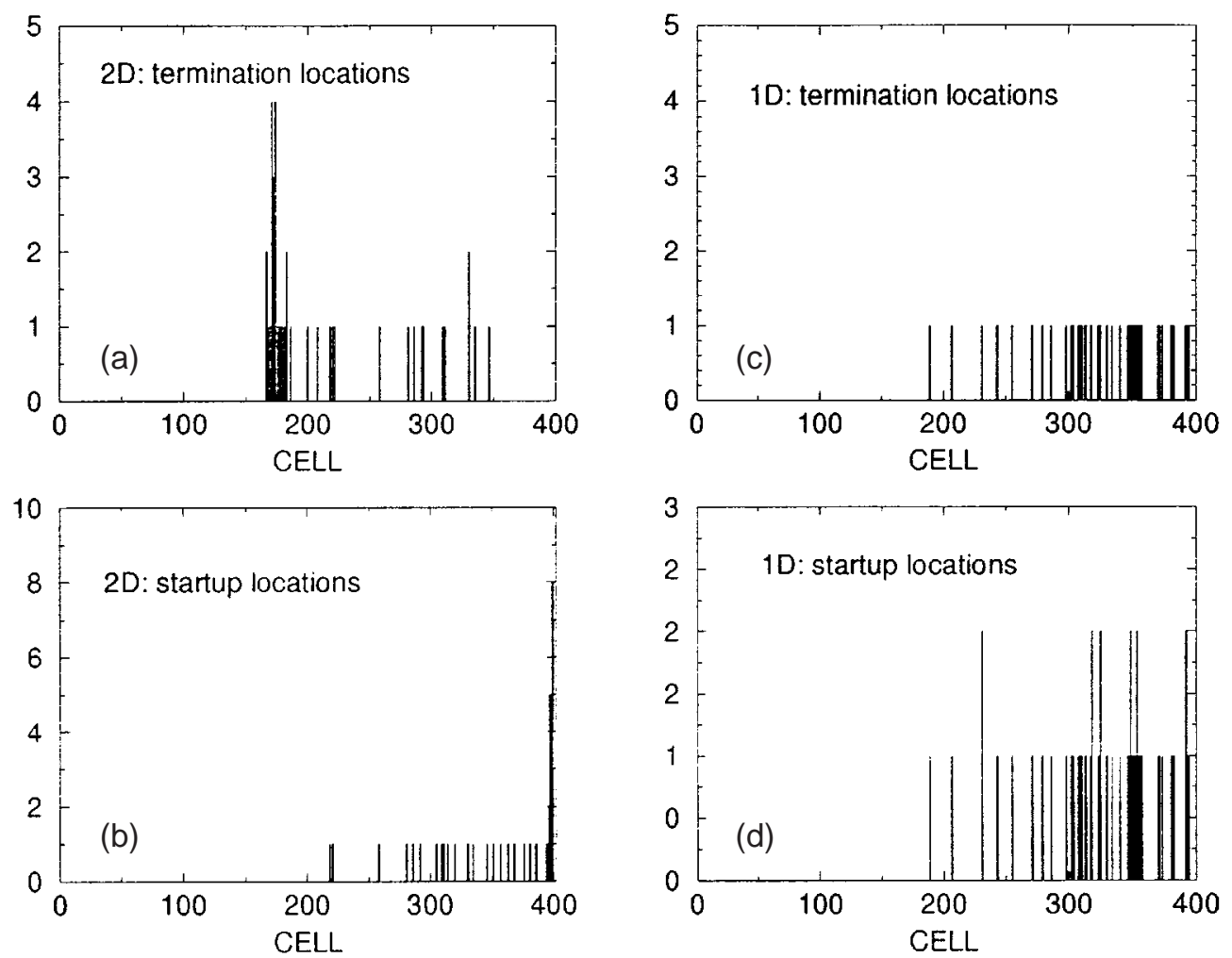

Figure 8. (a, c) Termination points and (b, d) PDF of avalanche startup for the diffusive sandpile case $\left(D_{0} / P_{0}=1\right)$.

system to stay in an SOC-like state, with avalanches of all sizes transporting energy and particles away from any unstable location in order to bring the profiles below the underlying instability threshold. However, no definitive experimental test to confirm this point is possible, since even the definition of what constitutes an SOC system is still a matter of discussion. In addition, some scepticism exists about the expectations of being able to test the system dynamics simply by taking edge measurements. As 
a consequence, a great controversy has developed within the fusion community, with different opinions existing on whether the available experimental evidence (basically, measurements of electrostatic fluctuations at the edge of several tokamaks and stellarators that exhibit long range time correlations and self-similarity down to scales of the order of ten times the turbulence decorrelation time) supports or invalidates the SOC model as a paradigm for plasma transport $[12,13,20]$.

We think that some of the results of this article could help resolve part of this controversy. It is clear from the sandpile simulations that, in a system with diffusion such as a confined plasma, the region of the system expected to behave in an SOC manner extends in from the edge, due to the boundary conditions there. Therefore, it seems to make a lot of sense to try to test for possible plasma SOC-like behaviour by probing the edge region, which is certainly fortunate considering the difficulty of taking measurements further inside the plasma.

At the same time, another relevant result of the sandpile calculations is that we have been able to identify the trends that the system would follow under a certain type of tests in which the relative importance of diffusive transport and avalanche transport changes. These tests could be carried out at the edge of tokamaks or stellarators, by artificially varying the relative intensities of both channels. A possible way of achieving this would be to take fluctuation measurements at different radial positions, to take advantage of their different local dependences. Another possible way (but a much more complicated one) would be to take measurements at a zero poloidal velocity radial location for a set of discharges in which the configuration properties could be changed in a controllable way (for instance, reducing the magnetic well at the edge, that effectively would increase the turbulence levels due to resistive pressure driven turbulence [21]). If this proposed SOC-diffusive paradigm applies, clear signatures of the interaction of the two channels should then reveal themselves from a statistical analysis of these edge fluctuation data. These signatures should include a monotonic reduction of the $1 / f$ region of the fluctuation power spectrum, the disappearance of logarithmic tails in the fluctuation autocorrelation function and the reduction and eventual disappearance of the linear region in the $R / S$ series analysis.

Finally, we would like to discuss what we think is the most important result of this article: the increasing relevance of the edge in SOC diffusive dynamics.
In a pure SOC system, the edge is not of particular significance. But in the case of an SOC diffusive system, and even for very low diffusion, we have found that the edge comes to play a dominant role in the transport dynamics, with more and more of the major transport events triggered from it. In addition, avalanches initiated at the edge penetrate very deeply into the system, reaching the core, and making the centre confinement properties strongly dependent on what is happening at the edge. The relevance of the edge may be the reason why core confinement in plasma confinement devices is so critically affected by good conditioning of the edge, as has been long known from many experiments. It could also probably shed some light on the role that neutrals might play as triggers for catastrophic avalanches. At the same time, it adds further confidence in making more edge fluctuation measurements in order to characterize the SOC behaviour of confined plasma. As a final comment, the steepest region extending inwards from the edge might constitute a natural definition of what the plasma edge is.

\section{Acknowledgements}

Valuable discussions with M. Varela, U.S. Bhatt, C. Hidalgo and P.H. Diamond are gratefully acknowledged. This research was supported in part by the Spanish DGES Project No. FTN2000-0924-C03-01 and Fundación Carlos III. This work has also been supported in part by the Office of Fusion Energy, USDOE, under Contract Nos DE-AC05-00OR22725 and DE-FG03-99ER54551.

\section{References}

[1] Bak, P., Tang, D., Weisenfeld, K., Phys. Rev. Lett. 59 (1987) 381.

[2] Shaw, B.E., Carlson, J.M., Langer, J.S., J. Geoph. Res. 97 (1992) 479.

[3] Bak, P., Sneppen, K., Phys. Rev. Lett. 71 (1993) 4083.

[4] Drossel, B., Schwabl, F., Phys. Rev. Lett. 69 (1992) 1629.

[5] Nagatani, T., Physica A 218 (1995) 1.

[6] Field, S., Witt, J., Nori, F., Phys. Rev. Lett. 74 (1995) 1206.

[7] Newman, D.E., Carreras, B.A., Diamond, P.H., Phys. Rev. Lett. A 218 (1996) 58.

[8] Diamond, P.H., Hahm, T.S., Phys. Plasmas 2 (1995) 3640. 


\section{R. Sánchez et al.}

[9] Carreras, B.A., et al., Phys. Rev. Lett. 80 (1998) 4438.

[10] Dendy, R.O., Helander, P., Plasma Phys. Control. Fusion 39 (1997) 1947.

[11] Politzer, P.A., Phys. Rev. Lett. 84 (2000) 1192.

[12] Carbone, V., Regnoli, G., Martines, E., Antoni, V., Phys. Plasmas 7 (2000) 445.

[13] Carreras, B.A., et al., Phys. Plasmas 7 (2000) 3278.

[14] Hinton, F.L., Hazeltine, R.D., Rev. Mod. Phys. 48 (1976) 240.

[15] Liewer, P.C., Nucl. Fusion 25 (1985) 543.

[16] Hwa, T., Kadar, M., Phys. Rev. A 45 (1992) 7002.

[17] Kadanoff, L.P., Nagel, S.R., Wu, L., Zhou, S.-M., Phys. Rev. A 39 (1989) 6542.

[18] Mandelbrot, B.B., Wallis, J.R., Water Resour. Res. 5 (1969) 967.
[19] Hurst, H.E., Trans. Am. Soc. Civ. Eng. 116 (1951) 770 .

[20] Krommes, J.A., Phys. Plasmas 6 (2000) 3731.

[21] Alejaldre, C., Review of confinement and transport studies in the TJ-II flexible heliac, Nucl. Fusion (in press).

(Manuscript received 6 October 2000

Final manuscript accepted 6 December 2000)

E-mail address of R. Sánchez:

rsanchez@fis.uc3m.es

Subject classification: F1, Gt 